\title{
Least Squares Estimation of Generalized Space Time AutoRegressive (GSTAR) Model and Its Properties
}

\author{
Budi Nurani Ruchjana*, Svetlana A. Borovkova ${ }^{\dagger}$ and H. P. Lopuhaa** \\ * Department of Mathematics, Faculty of Mathematics and Natural Sciences \\ Universitas Padjadjaran, Sumedang 45363, INDONESIA \\ ${ }^{\dagger}$ Department of Finance, Faculty of Economics, Vrije Universiteit Amsterdam, De Boelelaan \\ 1105, $1081 \mathrm{HV}$, Amsterdam, THE NETHERLANDS \\ ${ }^{* *}$ Faculty of EEMCS, Delft Institute of Applied Mathematics, Delft University of \\ Technology, Mekelweg 4, 2628 CD Delft,THE NETHERLANDS \\ budinr@unpad.ac.id
}

\begin{abstract}
In this paper we studied a least squares estimation parameters of the Generalized Space Time AutoRegressive (GSTAR) model and its properties, especially in consistency and asymptotic normality. We use R software to estimate the GSTAR parameter and apply the model toward real phenomena of data, such as an oil production data at volcanic layer.
\end{abstract}

Keywords: GSTAR, least squares, consistency, asymptotic normal

PACS: $02.50 .-\mathrm{r}$

\section{INTRODUCTION}

\subsection{The Generalized STAR Model}

The space time models such as Space Time Autoregressive (STAR) introduced by [2] and [6]. It appeared at about 1970s when [4] introduced the multivariate time series as an expansion for univariate time series model from [3]. These model are part of stochastic processes. Stochastic process is the set of a given sequence of random variables [5], for example the sequence of time, space, or a combination of space and time. The STAR model from [2] and [6] just only applicable for homogeneous locations, because all of locations have a same value of time series and space time parameters.

In this paper, we studied a least squares properties of an estimator parameters of the Generalized STAR (GSTAR) model, especially on consistency and asymptotic normality. The GSTAR model is proposed by [2] based on oil production phenomenon volcanic layer in Jatibarang Field, West Java Indonesia. The GSTAR model is a natural generalization of STAR models, allowing the autoregressive parameters to vary per location, so the GSTAR model is applicable for heterogeneous characteristic of sample locations. The $\operatorname{GSTAR}\left(\lambda_{k}, p\right)$ model is written as:

$$
\mathbf{z}(t)=\sum_{k=1}^{p} \sum_{l=0}^{\lambda_{k}} \Phi_{k l} \mathbf{W}^{(l)} \mathbf{z}(t-k)+\mathbf{e}(t)
$$

$$
\begin{aligned}
& \text { where, } \\
& \mathbf{z}(t) \quad: \quad(N \times 1) \text { of observation vector at time } t \\
& \lambda_{k} \quad \text { : spatial order of the } k^{\text {th }} \text { autoregressive } \\
& \text { term } \\
& \Phi_{k l} \quad \text { : the diagonal matrices with the diag- }
\end{aligned}
$$

The GSTAR $(1 ; 1)$ model which both of parameters $\Phi_{0}^{(i)}$ and $\Phi_{1}^{(i)}$ are changing is presented:

$$
\mathbf{z}(t)=\Phi_{0} \mathbf{z}(t-1)+\Phi_{1} \mathbf{W} \mathbf{z}(t-1)+\mathbf{e}(t)
$$

where the $\Phi_{0}$ is the diagonal matrices with the diagonal elements as the autoregressive parameters of lag time 1 for each location $\left(\Phi_{0}^{(1)}, \cdots, \Phi_{0}^{(N)}\right)$. The $\Phi_{1}$ is the diagonal matrices with the diagonal elements as the space time parameters in the lag spatial 1 and lag time 1 for each location $\left(\Phi_{1}^{(1)}, \cdots, \Phi_{1}^{(N)}\right)$. The GSTAR $(1 ; 1)$ can be written as the vector autoregressive order one model or $\operatorname{VAR}(1)$ :

$$
\begin{gathered}
\mathbf{z}(t)=\Phi \mathbf{z}(t-1)+\mathbf{e}(t) \\
\Phi=\left[\Phi_{10}+\Phi_{11} \mathbf{W}\right]
\end{gathered}
$$




\subsection{Least Squares Estimator of GSTAR(1;1)}

Consider the GSTAR $(1 ; 1)$ model defined through (2). Suppose we have a linear model for GSTAR:

$$
Y_{i}=X_{i} \beta+u_{i}
$$

where,

$$
\begin{gathered}
Y_{i}=\left[\begin{array}{c}
Z_{i}(2) \\
Z_{i}(3) \\
\vdots \\
Z_{i}(T)
\end{array}\right], X_{i}=\left[\begin{array}{cc}
Z_{i}(1) & V_{i}(1) \\
Z_{i}(2) & V_{(i)}(2) \\
\vdots & \vdots \\
Z_{i}(T-1) & V_{i}(T-1)
\end{array}\right], \\
u_{i}=\left[\begin{array}{c}
e_{i}(2) \\
e_{i}(3) \\
\vdots \\
e_{i}(T)
\end{array}\right]
\end{gathered}
$$

and $V_{i}(t)=\sum_{j=1}^{N} w_{i j} Z_{j}$, for $i \neq j$.

Consequently, the model equations for all locations simultaneously follow the linear model structure. Note that for each site $i=1,2, \cdots, N$ we have a separate linear model $\mathbf{Y}_{i}=\mathbf{X}_{i} \beta_{i}+\mathbf{u}_{i}$, which means that for each site the least squares estimator for $\beta_{i}$ can be computed separately. However, the value of the estimator does depend on the values of $Z_{i}(t)$ at other sites, because the form of $V_{i}(t)$.

For later theoretical purposes we bring in some additional structure to separate the deterministic weights $w_{i j}$ from the random variables $Z_{i}(t)$. If, for each $i=1,2, \cdots, N$, we define

$$
M_{i}=\left[\begin{array}{ccccccc}
0 & \cdots & 0 & 1 & 0 & \cdots & 0 \\
w_{i 1} & \cdots & w_{i, i-1} & 0 & w_{i, i+1} & \cdots & w_{i N}
\end{array}\right]
$$

then $\mathbf{X}_{i}$ can be written as

$$
\mathbf{X}_{i}=\mathbf{M}_{i}\left[\begin{array}{llll}
\mathbf{Z}(0) & \mathbf{Z}(1) & \cdots & \mathbf{Z}(T-1)
\end{array}\right]
$$

and we can wrote

$$
\mathbf{X}^{\prime}=\mathbf{M}\left(\mathbf{I} \otimes\left[\begin{array}{llll}
\mathbf{Z}(0) & \mathbf{Z}(1) & \cdots & \mathbf{Z}(T-1)
\end{array}\right]\right)
$$

where $\mathbf{M}=\operatorname{diag}\left(\mathbf{M}_{1}, \cdots, \mathbf{M}_{N}\right)$.

Here $\mathbf{A} \otimes \mathbf{B}$ denotes the block matrix with blocks $a_{i j} \mathbf{B}$. We conclude that the least squares estimator $\beta_{T}$ for $\beta$ satisfies the usual normal equations $\mathbf{X}^{t} \mathbf{X} \beta_{T}=\mathbf{X}^{\prime} \mathbf{u}$, with $\mathbf{X}$ and $\mathbf{u}$ described above, and can be determined uniquely as soon as the matrix $\mathbf{X}^{\prime} \mathbf{X}$ is nonsingular.

\section{PROPERTIES OF THE LEAST SQUARES ESTIMATOR OF GSTAR PARAMETER}

The model (2) can be seen as a multiple linear regression model with random covariates:

$$
y_{t}=\mathbf{x}_{t} \beta+\varepsilon_{t} \quad t=1,2, \cdots, n
$$

where $\beta$ is a $(k 1)$ vector of parameters, $\mathbf{x}_{t}=\left(x_{t 1}, \cdots, x_{t k}\right)$ is a $(1 k)$ vector of explanatory variables, and $\varepsilon_{1}, \varepsilon_{2}, \cdots, \varepsilon_{n}$ are random variables. The behavior of the least squares estimator in such models, in particular the behavior of

$$
\sum_{t=1}^{n} x_{t i} x_{t j} \text { and } \sum_{t=1}^{n} x_{t i} \varepsilon_{t} \text { for } i, j=1,2, \cdots, n
$$

has been of interest to many authors [7] (e.g. see Cristopeit and Helmes, 1980 and Lai and Wei, 1982, who also provide further references to the statistical and engineering literature; see also White, 1984, for references to the econometrics literature). Strong consistency and asymptotic normality of the least squares estimator is established by these authors under relatively mild conditions. However, their results do not directly apply to our situation.

In the GSTAR model, the sequence is formed by all $e_{i}(t)$ values, for $i=1,2, \cdots, N$ and $t=1,2, \cdots, T$ for which at a fixed time $t$, the elements $e_{1}(t), \cdots, e_{N}(t)$ of the error vector $\mathbf{e}(\mathrm{t})$ still can be correlated. We will assume a martingale difference array structure for the vectors $\mathbf{e}(\mathrm{t})$, but this does not imply a similar structure for the entire sequence of individual components $e_{i}(t)$. White (1984) in [7] treats linear regression models with matrixvalued $\mathbf{x}_{t}$ and vector-valued $\varepsilon_{t}$, but requires either mixing, stationary ergodicity, or asymptotic independence of the sequences $\left\{\mathbf{x}_{t}\right\}$ and $\left\{\varepsilon_{t}\right\}$. This is stronger than necessary in our specific setup, where we only Alternatively, one may attempt to apply existing results for vector autoregressive models, since the GSTAR model (2) can be written as a VAR model.

We use the specific structure of the matrix $\mathbf{A}=\Phi_{0}+$ $\Phi_{1} \mathbf{W}$ directly and formulate the GSTAR model as a linear model in such a way as to avoid the 'restricted LS reasoning'.

The properties of consistency and asymptotic normality of least squares estimator of GSTAR has been proved [7]. We assume that the observations are centered, i.e. $E[\mathbf{Z}(t)]=0$ and that:

(C1) the characteristic roots of the matrix $\Phi_{0}+\Phi_{1} \boldsymbol{W}$ are less than one in absolute value.

Assumption (C1) ensures that the $\operatorname{GSTAR}(1 ; 1)$ model has a causal stationary solution. We establish the statistical properties of the least squares estimator under 
the assumption that the sequence $\{e(t)\}$ forms a vectorvalued dmartingale difference array with respect to an increasing sequence of C-fields $\left\{F_{t}\right\}$, i.e.

(C2) $\mathbf{e}(t)$ is $F_{t}$-measurable and $E\left[\boldsymbol{e}(t) \mid F_{t-1}\right]=\boldsymbol{0}$.

A similar setup is considered by Lai and Wei (1982) and White (1984) in linear regression models, and by Anderson and Taylor (1979), Duflo et al. (1991), and Anderson and Kunitomo (1992) in VAR models [7]. Because condition (C1) implies causality of the solution of (2), i.e. $\mathbf{Z}(t)$ is a linear combination of the current and past disturbances $\mathbf{e}(s), s \leq t$, it suffices to assume moment conditions on $\mathbf{e}(t)$ only. We assume:

(C3) $E\left[\mathbf{e}(t) \mathbf{e}(t)^{\prime} \mid F_{t-1}\right]=\sum_{t}$ a.s, $T^{-1}=\sum_{1}^{T} \sum_{t}$ converges in probability to a constant matrix $\sum$.

(C4) $\sup _{t \geq 1} E\left[\mathbf{e}(t)^{t} \mathbf{e}(t)\left\{\mathbf{e}(t)^{t} \mathbf{e}(t)>a\right\} \mid F_{t-1}\right]$ converges to zero in probability, as a goes to infinity.

Finally, we define

$$
\Gamma=\sum_{j=0}^{\infty} A^{j} \sum\left(A^{\prime}\right) \quad \text { where } A=\Phi_{0}+\Phi_{1} \mathbf{W}
$$

Theorem 1 establishes weak consistency of the least squares estimator, the proof is given at [7].

Theorem 1 Let $\beta=\left(\phi_{01}, \phi_{11}, \phi_{02}, \phi_{12}, \cdots, \phi_{0 N}, \phi_{1 N}\right)^{\prime}$ be the vector of parameters in the GSTAR $(1 ; 1)$ model and let $\Gamma$ be defined in (10). If $\Gamma$ is nonsingular, then under conditions $(C 1)-(C 4)$, the least squares estimator $\beta_{T}$ converges to $\beta$ in probability.

Asymptotic normality can be established under conditions (C1)-(C4) and

(C5) For all $r, s=1,2, \cdots, N$

$$
T^{-1} \sum_{t=\max , s+2}^{N} \sum_{t} \otimes \mathbf{e}(t-1-r) \mathbf{e}(t-1-s) .
$$

Theorem 2 Let $\beta=\left(\phi_{01}, \phi_{11}, \phi_{02}, \phi_{12}, \cdots, \phi_{0 N}, \phi_{1 N}\right)^{\prime}$ be the vector of parameters in the GSTAR $(1 ; 1)$ model, let $\beta_{T}$ be the corresponding least squares estimator, and let $\Gamma$ be as defined in (10). If $\Gamma$ is nonsingular, then under conditions $(C 1)-(C 5), \sqrt{ } T\left(\beta_{T}-\beta\right)$ converges in distribution to a $2 N$-variate normal distribution with mean zero and covariance matrix

$$
\left(M(I \otimes \boldsymbol{r}) M^{\prime}\right)^{-1} M(I \otimes \boldsymbol{r}) M^{\prime}\left(M(I \otimes \boldsymbol{r}) M^{\prime}\right)^{-1}
$$

where $M=\operatorname{diag}\left(M_{1}, M_{2}, \cdots, M_{N}\right)$ with $M_{i}$ defined in (5).

\section{CASE STUDY}

For case study we use oil production data at volcanic layer Jatibarang, West Java Indonesia for 60 monthly data set for 3 oil wells. Because the volcanic is a heterogeneous layer, so the GSTAR model is suitable for this dataset. We built script file using $\mathrm{R}$ software to estimate the parameter of GSTAR $(1 ; p)$. Part of our programming is following command in R or S-Plus software:

GSTARfit<-function $(z, W, p)\{$

\# $\quad \mathrm{z}$ is a matrix containing a multivariate time series

\# columns are locations; rows are time

\# $\quad \mathrm{W}$ is a weight matrix; rowsums are 1; diagonal contains only zeros

\# $\mathrm{p}$ is the temporal order

N.obs<-nrow $(\mathrm{z}) \#$ note that rows $1,2, \ldots$, n.obs correspond to times $0,1,2, \ldots$, n.obs- 1

$\mathrm{N} . \operatorname{loc}<-\operatorname{ncol}(\mathrm{z})$

\#

\# $\quad$ preparation of lists

$\#$

$\mathrm{y}<-$ list()

$\mathrm{X}<-$-list ()

beta $<$-list ()

Phi0<-list() \# list of diagonals of matrices phi_s0 for each time lag $\mathrm{s}=1,2, \ldots, \mathrm{p}$

Phi1<-list() \# list of diagonals of matrices phi_10 for each time lag $\mathrm{s}=1,2, \ldots, \mathrm{p}$

1s.fit<-list() \# list of ls.fit for each linear model of the ith location

se.beta.ls<-list()

\# centering the input series

z.bar<-t(matrix $(\operatorname{colMeans}(\mathrm{z}), \mathrm{ncol}=\mathrm{N} . \mathrm{obs}, \mathrm{nrow}=\mathrm{N} . \mathrm{loc})$ )

cz<-z-z.bar

\# computing vectors $\mathrm{v}(\mathrm{t})=\mathrm{wz}(\mathrm{t})$

$\mathrm{cV}<-\mathrm{t}(\mathrm{W} \% * \% \mathrm{t}(\mathrm{cz}))$

\#

\# setting up the linear model for gstar and compute ls estimates

Etc.

For running the script file, we use command:

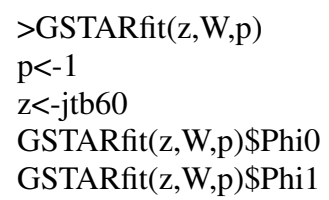

$>$ W

\#spatial weight matrix

$\begin{array}{lccc} & {[, 1]} & {[, 2]} & {[, 3]} \\ {[1,]} & 0.000 & 0.605 & 0.395 \\ {[2,]} & 0.657 & 0.000 & 0.343 \\ {[3,]} & 0.566 & 0.444 & 0.000\end{array}$


And then we got the least squares estimator of GSTAR(1;1) as following Table 1.

Table 1. Least Squares Estimator of GSTAR(1;1)

\begin{tabular}{|c|c|c|c|}
\hline Well & $\phi_{0}^{i}$ & $\phi_{1}^{i}$ & AIC \\
\hline Well1 & 0.614 & 0.247 & \\
Well2 & 0.609 & 0.165 & 755.231 \\
Well3 & 0.810 & -0.034 & \\
\hline
\end{tabular}

For the GSTAR $(1 ; 2)$ means the model with the first lag in space and the second lag in time, we have the result as Table 2 .

Table 2. Least Squares Estimator of $\operatorname{GSTAR}(1 ; 2)$

\begin{tabular}{|c|c|c|c|c|c|}
\hline Well & $\phi_{10}^{i}$ & $\phi_{11}^{i}$ & $\phi_{20}^{i}$ & $\phi_{21}^{i}$ & AIC \\
\hline Well1 & 0.486 & 0.088 & 0.118 & 0.221 & \\
Well2 & 0.590 & 0.003 & -0.001 & 0.256 & 764.665 \\
Well3 & 0.542 & -0.084 & 0.332 & -0.0053 & \\
\hline
\end{tabular}

From above example we can explain that the leasts squares estimator of the $\operatorname{GSTAR}(1 ; 1)$ and $\operatorname{GSTAR}(1 ; 2)$ using spatial weight have a fit the oil production model, which the GSTAR $(1 ; 1)$ model has a minimum AIC. For both of GSTAR models there are significantly differences in parameters values at different locations. For all pairs of locations, we tested the parameters. The estimator of GSTAR are significantly different to zero.

\section{SUMMARY}

In this paper we studied the least squares estimator of GSTAR model. The estimator has properties of consistensy and asymptotic normality through the Theorem 1 and Theorem 2.

We built a script file using $\mathrm{R}$ software to estimate the parameter using least squares method. It should be done, because not yet space time software which can be used to estimate the parameters.

For case study to apply the GSTAR model to oil production data, we can summaries that the choice of temporal lag is more interesting than the choice of spatial lag.

\section{REFERENCES}

1. B. N. Ruchjana, (2002), The Generalized Space Time Autoregressive Order One Model and Its Application to Oil production Data, Unpublished Dissertation, Department Mathematics, Institut teknologi Bandung, Bandung.

2. A. D. Cliff and J. Ord, (1975), Model building and the analysis of spatial pattern in human geography,
Journal of the Royal Statistical Society, Series B 37, 297-348.

3. G. E. P. Box and G. M. Jenkins, (1976), Time series analysis: forecasting and control, Holden- Day Inc. San Francisco.

4. Hannan, E. J. (1970), Multiple time series, John Wiley \& Sons, New York.

5. N. Cressie, (1993), Statistics for Spatial Data, John Wiley \& Sons., Inc., New York.

6. P. E. Pfeifer and S. J. Deutsch, (1980), A three stage iterative procedure for space-time modeling, Technometrics 22, 35-47.

7. S. Borovkova, H. P. Lopuhaa, and B. N. Ruchjana, (2008), Consistency and Asymptotic Normality of Least Squares Estimators of Generalized STAR models, Statistica Neerlandica, 62, nr 4, p. 482-508. 